\title{
Spatially Resolved Velocity Mapping of the Melt Plume During High-Pressure Gas Atomization of Liquid Metals
}

\author{
T.D. BIGG and A.M. MULLIS
}

\begin{abstract}
We present details of an image analysis algorithm designed specifically to determine the velocity of material in the melt plume during high-pressure, close-coupled gas atomization. Following high-speed filming (16,000 fps) pairs of images are used to identify and track dominant features within the plume. Due to the complexity of the atomization plume, relatively few features are tracked between any given pair of images, but by averaging over the many thousands of frames obtained during high-speed filming a spatially resolved map of the average velocity of material in the plume can be built up. Velocities in the plume are typically very low compared to that of the supersonic gas, being around $30 \mathrm{~m} \mathrm{~s}^{-1}$ on the margins of the plume where the melt interacts strongly with the gas and dropping to $<10 \mathrm{~m} \mathrm{~s}^{-1}$ in the center of the melt plume. Consequently, the efficiency of the atomizer in transferring kinetic energy from the gas to the melt is correspondingly very low, with this being estimated as being no more than $0.1 \mathrm{pct}$.
\end{abstract}

https://doi.org/10.1007/s11663-020-01899-5

(C) The Author(s) 2020

\section{INTRODUCTION}

GAS atomization of molten metal is an important industrial process used to produce highly spherical metal powders for a range of industrial uses. The applications for such powders are diverse and include use as a feedstock for additive manufacturing, the production of catalysts for chemical processing, and formulation of brazing pastes for joining materials. Additive manufacturing in particular is creating both a massively increased demand for metal powders and a drive for improved powder quality (see, e.g., the recent review of powders for ALM feedstock by Anderson et al. $){ }^{[1]}$ For each application, the metal powder must meet a defined specification with one of the principal metrics employed being the particle size distribution (PSD) of the powder. Precise control of the gas atomization process is desirable in order to constrain the PSD produced, maximizing the usable fraction of powder and thereby minimizing scrappage or recycling of powder that is outside of the required specification. However, as noted by Anderson and Terpstra, ${ }^{[2]}$ the PSD of powders produced by gas atomization tends to be quite broad, typically spanning an order of magnitude or more,

T.D. BIGG and A.M. MULLIS are with the School of Chemical \& Process Engineering, University of Leeds, Leeds LS2 9JT, UK. Contact E-mail: t.bigg@leeds.ac.uk.

Manuscript submitted July 30, 2019.

Article published online July 17, 2020. leading to re-melt rates in commercial production that may be as high as 65 pct. Consequently, even modest improvements in control of the PSD could result in significant cost saving.

In the gas atomization process, the PSD of the powder produced is influenced by various physical processes. One of the most significant factors controlling the PSD is the way in which the jet or film of molten metal interacts with, and is broken up by, the gas stream during primary atomization. This in turn affects the way in which the molten metal droplets formed during primary atomization interact downstream with the gas during secondary atomization. Both primary and secondary break-ups are strongly influenced by gas and particle velocities and the resultant shear forces generated. Duke and Honnery ${ }^{[3]}$ have studied the position and velocity of the liquid-gas interface at the point where a liquid sheet becomes unstable prior to break-up into ligaments and droplets, finding that the Reynolds number, Weber number, and the gas/liquid momentum ratio were key parameters. Zandian et al. ${ }^{[4]}$ used the level sets method to investigate primary break-up of a liquid sheet by a high-pressure gas jet, demonstrating that both the Reynolds and Weber numbers* are key

*The differential velocity between the gas and the melt appear as a linear factor in the Reynolds number and a quadratic factor in the Weber number.

parameters in determining the mode by which such break-up occurs. Similarly, $\mathrm{Li}$ and Fritsching ${ }^{[5]}$ 
demonstrated that the drag force between the fast moving gas and the slower moving droplets was a key parameter in determining secondary break-up.

The break-up modes operating in turn determine the atomization efficiency, which is generally accepted as being very low. In a recent evaluation of greenhouse emissions related to powder metallurgy by Azevedo et al., ${ }^{[6]}$ a figure of $\sim 11$ pct is quoted, this being based upon the ratio of the actual energy used by the process to that used in some ideal theoretical process with minimum energy consumption. However, while such a basis may be useful in comparing the impact of different metallurgical processes, it is perhaps of less use when comparing detailed modifications within a process such as gas atomization. An alternative way of quantifying the efficiency of the atomization process is to consider the theoretical efficiency for the conversion of kinetic energy into the embodied surface energy of the powder. As shown by Yule and Dunkley, ${ }^{[7]}$ this usually results in very small values, typically $<0.01 \mathrm{pct}$, although such considerations were utilized by Strauss and Miller ${ }^{[8]}$ to develop, with some success, a physically based model for estimating the particle size during gas atomization.

Improving the efficiency of the gas atomization process has been an area of active research: the replacement of the conventional annular slit gas delivery system with discrete jet nozzles was suggested by Anderson and Terpstra, ${ }^{[2]}$ and the inclusion of resonant cavities to produce ultrasonic frequencies to aid ligament disintegration was patented by Grant. ${ }^{\left[{ }^{9]}\right.}$ However, modifications have not been limited to the gas delivery manifold. A slotted melt delivery nozzle was proposed by Anderson et al., ${ }^{[10]}$ in order to direct the melt towards the atomizing gas jets, while a CFD study of various melt nozzle designs by Motaman et al. ${ }^{[11]}$ demonstrated that the wake-closure pressure could be dramatically reduced by adding a curved concave profile to the inside of the melt nozzle. The nature of the atomizing gas and its temperature have also been the subject of investigation, with Dunkley et al. ${ }^{[12]}$ demonstrating significantly finer median particle size with reduced gas consumption if the gas feed is pre-heated to around $250{ }^{\circ} \mathrm{C}$.

However, much of the research into gas atomization has been conducted on a trial and error basis, examining the change in the PSD resulting from various design changes. This is, at least in part, because very few tools exist to monitor the gas atomization process in situ. The lack of such tools both limits the rate at which progress towards more efficient atomization can be realized and means that, even where progress is made, the underlying physical mechanisms operating may not be appreciated. In this research, we contend that, given the important role of shear forces in liquid break-up, obtaining spatial mapping of the velocity distribution within an atomization melt plume could help to understand the reasons for this low efficiency and could potentially be a useful tool to drive efficiency improvements within the industry. Moreover, such a spatial mapping of the velocity within the melt plume can be used to arrive at much more direct measures of the efficiency for a particular atomizer design. Once the velocity distribution is known, it is a relatively straightforward matter to calculate the kinetic energy (or momentum) embodied within the melt plume by virtue of its motion. This may in turn be compared to the kinetic energy (or momentum) embodied within the gas stream, which may be calculated via isentropic flow theory. Consequently, a very direct measure of efficiency may be defined and evaluated for a given atomizer design, namely the efficiency of transferring kinetic energy (or momentum) from the supersonic gas stream to the melt plume.

One method that can be used to obtain velocity metrics from the gas atomization process is to employ high-speed photography to record images of the gas atomization plume. One such estimate, based on a single feature and at a single instant in time, is given by Mullis et al., ${ }^{[13]}$ wherein they estimated the velocity (or more specifically the component of the velocity in the plane of the image) of a group of co-moving particles within a plume as being around $30 \mathrm{~m} \mathrm{~s}^{-1}$. However, most high-speed imaging applied to gas atomization has been used either to study instabilities within the process, as has been done by Mullis et al., ${ }^{[14]}$ or to infer properties of the gas dynamics, such as Mates and Settles's ${ }^{[15]}$ study of wake-closure.

Object tracking is widely used in other fields to determine the velocity of objects as they cross the field of view of a camera, with an extensive literature available on both Fourier (see, e.g., Ejiri and Hamada ${ }^{[16]}$ ) and non-Fourier (see, e.g., Malavika and Poornima $^{[17]}$ )-based techniques. For example, pre-recorded or real-time images can be used to track the movement of objects, such as vehicles on a highway. In the case of vehicle tracking, it is considered that the objects being tracked present a relatively regularly shaped object, albeit slowly changing due to perspective as the camera-to-vehicle distance and viewing angle changes. Conversely, in a gas atomization plume, the features being observed are highly fluid in that particles can be seen forming dense clouds which are then observed to move, rapidly change shape, and disperse. The highly fluid nature of the gas atomization process means that the observable features may have either hard edges or relatively diffuse borders. Moreover, as gas atomization is typically filmed using the radiant light from the hot molten metal, cooling of the atomized droplets in flight means that the illumination of the features changes rapidly. Furthermore, using a two-dimensional recording system to analyze a three-dimensional process also adds to the complexity. In a vehicle tracking application, it is considered that using a suitably elevated camera position for capturing images mitigates against problems caused by using a two-dimensional image capture system to analyze a three-dimensional situation. However, in a gas atomization plume it is inevitable that some features will be obscured and that the images recorded will include instances where two or more features located at different distances from the camera appear as a single feature, and so cannot be differentiated from each other. Consequently, when conventional object tracking routines are applied to an atomization plume, they tend to perform very poorly. 
Although there are clearly challenges involved in using images from high-speed photography to obtain quantitative information about the gas atomization process, when viewing such high-speed camera images, it is possible to readily discern features that persist in a recognizable form from one image to the next. As would be expected, the motion of these features usually indicates a direction of travel away from the melt ejection nozzle. However, close to the nozzle an area of recirculation exists and features are sometimes observed traveling towards the nozzle. The failure of conventional object tracking routines appears to arise from (i) there being no clear distinction between the object to be tracked and the background and (ii) the variability of the objects being tracked.

A number of researchers (e.g., Mullis et al. ${ }^{[18]}$ Kirmse and Chaves ${ }^{[19]}$ Planche et al., ${ }^{[20]}$ and Pham et al. $\left.{ }^{[21]}\right)$ have used particle image velocimetry (PIV) to try and estimate the velocity of particulate material in an atomization process. This technique uses two short pulses from a laser to illuminate a thin sheet or slice of the atomization plume a short time duration apart. The velocity estimation is obtained by using PIV evaluation software to determine the displacement of particles over the time interval between the two laser pulses. However, the flow of the second fluid (melt or tracer particles) has to be very low in such experiments, wherein the atomizer is close to operating in gas-only flow. Consequently, the measured flow field is not representative of coupled flow at a realistic gas-to-metal ratio. Duke and Honnery ${ }^{[3]}$ have reported an experimental method for investigating the break-up of a two-phase flow, using a cross-correlation technique, although this was applied to a liquid sheet and may therefore be unsuitable to the chaotic motion within a gas atomization plume. Outside of the field of gas atomization, Tokumaru and Dimotakis ${ }^{[22]}$ have estimated flow velocities in single-phase liquid flows and single-phase gaseous flows using a method for transforming images of flows. By using Taylor series expansions of the Langrangian displacement field, they have demonstrated that it is possible to correlate two successive images for a range of transformations, and from this obtain vector fields indicating flow velocity and direction.

In this paper, we present the development of a specially designed computer vision algorithm designed to build up a two-dimensional spatial map of particle velocities in the atomization plume. Rather than the deterministic approach used in conventional object tracking in which well-delineated objects move from frame to frame with little variation in form, we use a statistical approach. We first search for the most distinctive features within a frame and then attempt to find a match for them in the next, accepting that in many, and possibly the majority, of cases such a match between frames will not exist. In fact, most image pairs contain only a relatively small number of trackable features and consequently many thousands of image pairs are required in order to build up the complete two-dimensional spatial map of the velocity profile within the gas atomization plume. The result is a time-averaged, spatially resolved map of the velocity of the second fluid (i.e., the metal) during gas atomization. Such a map can help us to elucidate the physical processes by which melt disintegration occurs, understand the low efficiency encountered in gas atomization processes, and act as a quantitative data set for the validation of CFD models of the atomization process.

\section{METHOD OF IMAGE CAPTURE}

Images of the gas atomization plume were captured during atomization of $316 \mathrm{~L}$ stainless steel. The melt was subject to a $200 \mathrm{~K}$ superheat, wherein the pour temperature was $\sim 1900 \mathrm{~K}$. Atomization was via an annular slit type atomizer operating at $2.6 \mathrm{MPa}$. The melt nozzle is of the conventional truncated cone type, with a $30 \mathrm{deg}$ apex angle, a 9-mm-diameter flat tip, and a central melt feed tube of $5 \mathrm{~mm}$ in diameter. The melt and gas flow rates were 0.25 and $0.35 \mathrm{~kg} \mathrm{~s}^{-1}$, respectively.

A Photron FastCam Mini UX100 High Speed Camera operating at 16,000 frames per second was used to image the melt plume, with a total of 28,665 frames being captured giving a total recording time of 1.7916 seconds. At this frame rate, the standard UX100 frame size is $1280 \times 312$ (width $\times$ height) and in order to accommodate the geometry of the atomization plume during vertical atomization the camera was mounted on its side. The subsequent images have been rotated so that they depict atomization in the direction in which it occurred (vertically downwards) and cropped to $312 \times 800$ to remove extraneous material. Each pixel is recorded in an 8-bit format, giving a 0 to 255 grayscale. The camera was equipped with high magnification micro-Nikkor optics which allowed full frame images to be obtained at a working distance of $\sim 30 \mathrm{~cm}$. The effective resolution with this setup is $\sim 0.0866 \mathrm{~mm}$ pixel $^{-1}$.

\section{UNDERLYING ASSUMPTIONS OF THE COMPUTER VISION ALGORITHM}

To carry out this type of image analysis and derive quantitative information about the atomization process, it was necessary to make some assumptions. Firstly, it was assumed that the atomizing gas and atomized material moves in only one dimension. The assumption is that the atomizing gas and atomized material moves only away from or towards the melt nozzle, i.e., vertically downwards or vertically upwards in the high-speed camera images presented herein. A small recirculation zone may exist close to the melt nozzle, which can be theorized to be a region consisting of a ring vortex (toroidal vortex), and so this assumption is not strictly correct, as in order for recirculation to occur a flow of atomizing gas and atomized material must exist in more than one dimension. However, with the exception of the small recirculation zone close to the nozzle, we consider that the flow of atomizing gas and atomized material is mainly one dimensional, and so this is not an unreasonable assumption to make. By making this assumption, the computer vision algorithm is greatly simplified. 
Secondly, it was assumed that a two-dimensional monitoring technique (high-speed camera) can be used to obtain quantitative information from a three-dimensional process. As has already been mentioned in the introduction, using a monitoring technique that records in two dimensions to investigate a process that operates in three dimensions is sub-optimal, as the presence of atomized material may be obscured by atomized material that is closer to the high-speed camera. In the recirculation zone close to the nozzle, the existence of a ring vortex here would mean that the flow vectors at this location are likely to be significantly more three-dimensional than at other locations further downstream. However, inspection of high-speed camera images of the atomization process suggests that the process is somewhat chaotic, and that the kind of stable flow pattern seen in gas-only CFD models of the atomization process does not form. The chaotic nature of the process means that it is possible to observe and track discrete clouds of atomized material, despite the limitations of using a two-dimensional recording process to investigate a three-dimensional process.

Thirdly, when estimating the total amount of kinetic energy contained within the plume of atomized material, it is assumed that the mass of atomized material present within each pixel of the image is proportional to the brightness (grayscale value) of that pixel. As the sole source of illumination for the high-speed photography is the incandescence of the atomized material, and the atomized material is on average cooler, and therefore less bright, further away from the melt nozzle, a correction is applied to the grayscale values. The correction that was made is contained within the description of the experimental method.

Fourthly, to assist in the estimation of kinetic energy within the plume of atomized material, it is assumed that when averaged over a sufficiently large number of frames, the mass flow rate of atomized material, although seemingly somewhat chaotic and highly variable over the timescales that are resolved by 16,000 frames per second imaging, can be assumed to be in a relatively steady state when averaged over the full 28,665 frames $(1.7916 \mathrm{~s})$ that are analyzed.

Lastly, it is assumed that when averaged over the full 28,665 frames $(1.7916 \mathrm{~s})$, horizontal slices of the atomization plume contain an equal mass of atomized material, irrespective of the displacement of the horizontal slice relative to the melt nozzle. This assumption builds on the fourth assumption by applying conservation of mass considerations and assuming that atomized material is, on average, steadily proceeding vertically downwards away from the melt nozzle.

\section{METHOD OF ESTIMATING VELOCITY FROM SEQUENTIAL PAIRS OF IMAGES}

A computer vision algorithm was created within MATLAB to perform analysis on sequential pairs of images, which for simplicity we will refer to as image 1 and image 2 . Here we give a generic overview of the algorithm, with a more technical description of the process including all the computational parameters used and the values they are assigned being given in Appendices $\mathrm{A}$ to $\mathrm{F}$.

The process begins in the top left-hand corner of image 1. We scan downwards through the first column of pixels (this will correspond to the dominant flow direction) looking for a grayscale density gradient (dark to bright) which exceeds some preset threshold. Once such a threshold is detected the search continues downwards to find the location of the maximum gradient. This location is marked as being the leading edge of a feature which may potentially be trackable in image 2. The process then continues in image 1 moving downwards through each column and left-to-right across the columns until all columns have been scanned. The output at this stage is a set of marked locations within image 1 that correspond to the most prominent dark-to-light transitions when scanning downwards through the image. By viewing a large sample of images, we judge that this process is reasonably robust and a good approximation to how a human viewer would manually identify dominant features within the atomization plume. The set of features identified in a particular frame 1 is shown in Figure 1(a).

The next stage in the process is to attempt to find these features in image 2. For each feature, the search in image 2 begins at the same location as that at which the feature was identified in image 1 . We search upwards and downwards (upwards to allow for recirculation effects near the nozzle, and downwards as this is the dominant flow direction) to see if the same feature can be identified. Initially we search in an upwards direction, and if a density gradient is identified we perform a check to try to establish if this is the same feature. This check is based either on the magnitude of the two density

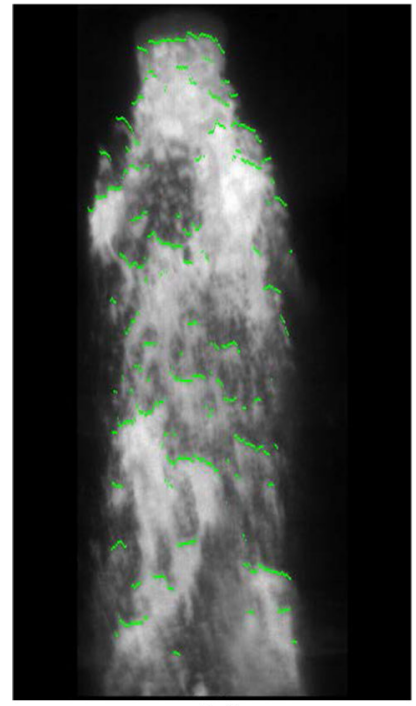

(a)

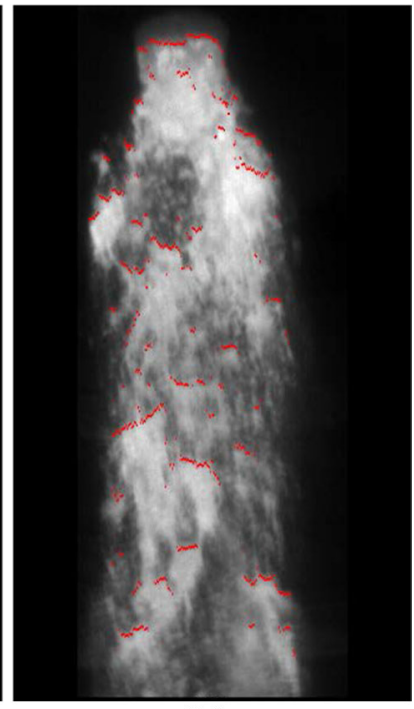

(b)
Fig. 1-An example of the analysis of an image pair (a) with locations of all the local maxima in the grayscale density gradient identified by the computer vision algorithm highlighted in green in image 1 and (b) with the new locations of density gradients previously identified highlighted in red in image 2 (Color figure online). 


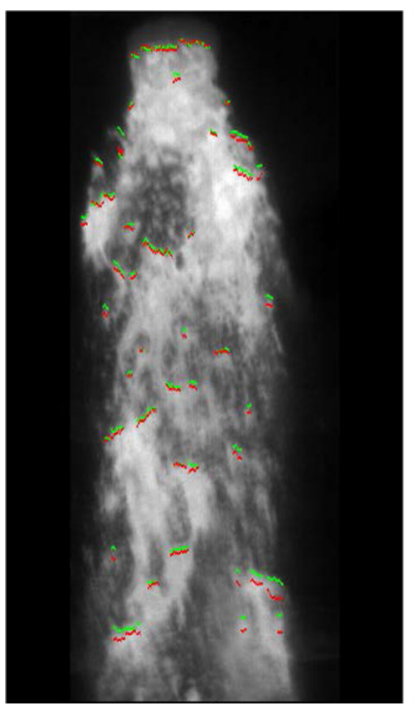

(a)

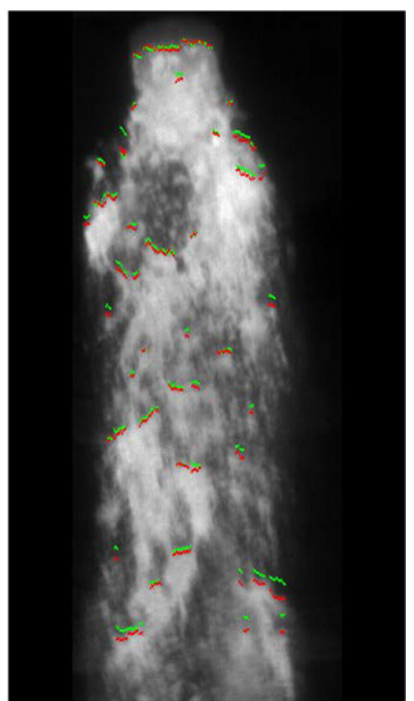

(b)

Fig. 2-Examples of images analyzed as image $1(a)$ and image $2(b)$ after filtering for minimum density gradient feature width and maximum allowable vertical step between neighboring density gradients. Density gradient locations identified in image 1 are highlighted in green, and new locations in image 2 of density gradients previously identified in image 1 are highlighted in red (Color figure online).

gradients being similar, to within some tolerance, or by calculation of the correlation coefficient between the two local regions in images 1 and 2, which is again compared to some tolerance. If the match is accepted, we mark the feature as having been tracked from image 1 to image 2, noting the negative displacement such that a negative velocity can be determined. If the match is not accepted, we continue to scan, although a maximum extent on the upwards search is set as features which have a very large displacement between frames are likely to result in a false match. After scanning in the upwards direction, a downwards search is instigated, using essentially the same criteria. Again, a maximum search extent is set, which may be different to the maximum extent in the upwards direction. If a match is found, a positive displacement for the feature is recorded, if no match is found within the upwards and downwards search ranges, then the feature has been 'lost' and it is assumed it is not trackable between frames 1 and 2. If more than one location meets the defined tolerance criteria, then we accept the match for the location in image 2 that has the closest density gradient or highest correlation coefficient to the feature found in image 1 . The set of trackable features between two consecutive images is shown in Figure 1(b).

The final stage in the process performs a set of sanity checks to increase the robustness of the identification and tracking of features. There are two distinct criteria that we apply, the first relates to the length of the identified feature, the second the size of any vertical jumps while traversing the length of a feature. Consider the latter of these first and say for the sake of argument that a maximum density gradient has been identified in column 1 that is located in row 20. If in column 2 a

maximum density gradient is also identified in row 20 , it is very likely these belong to the same feature, whereas if the nearest maximum density gradient is in say row 50, it is quite unlikely that this belongs to the same feature. This of course is an extreme example and it is much more likely that the vertical offset between columns is only a few pixels. Consequently, some tolerance has to be set on this maximum vertical offset to allow us to distinguish between a single feature that might be inclined to the horizontal (or curved) and two nearby features. If this tolerance is exceeded, the feature is split into two, if it is not it is considered to be all the same feature. The other condition relates to the length of the resulting features. A feature that is only 1 or 2 pixels long is much more likely to be an artifact than one that can be traced across say 20 consecutive columns in the image. Consequently, any feature that does not meet a minimum length requirement is discarded. The result at the end of this final identification stage is a set of features which have been tracked between image pairs and in which we have reasonable confidence that the feature in image 2 is the same one as identified in image 1 .

At the end of this identification process, the result is a set of features that have been tracked between images. As can be seen in Figures 2(a) and (b), this is a relatively sparse set. We therefore move along the image set, with image 2 now becoming image 1 and the next in the sequence becoming image 2 , with this process being repeated until all images in the movie have been processed. In order to create a fully populated spatial map, the data from all the image pairs analyzed were combined, wherein various metrics including average and RMS displacement, together with 5th percentile and 95th percentile displacement and other statistics, related to the analysis method, as a function of spatial position can be calculated. Moreover, as both the time-base between images and the absolute size in the image plane per pixel are known, the observed displacement of tracked features between image pairs may be converted from a pixel displacement to a velocity estimation. Graphical outputs were created by overlaying these maps onto an average image of the atomization plume. The average image of the atomization plume was derived by summing the grayscale intensities from all the images analyzed and dividing by the number of images analyzed. In this way, a spatially resolved average velocity for each location in the movie can be built up, with this essentially being the final output of the analysis.

In a computer vision algorithm such as this, it is inevitable that some false matches will occur in image 2. False matches occur when the computer vision algorithm incorrectly tracks a density gradient to a new position in image 2 . Situations such as this arise because the algorithm cannot fully account for the complex nature of the process and because of the limitations of the recording mechanism used. In particular, as has already been mentioned, the features being tracked are highly fluid clouds of particles that can rapidly change shape, and become more or less dense. Recording limitations occur because in a two-dimensional 


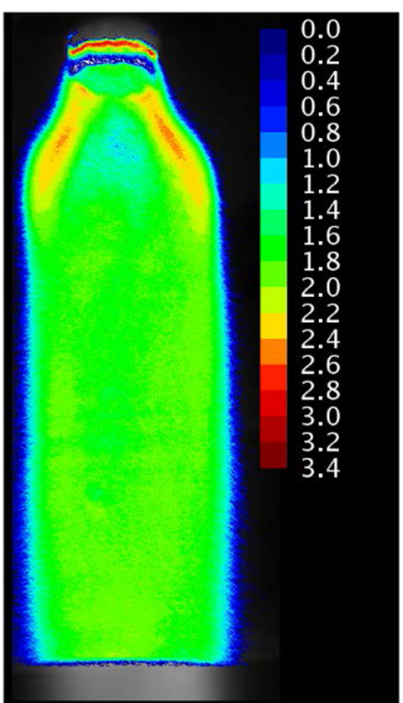

(a)

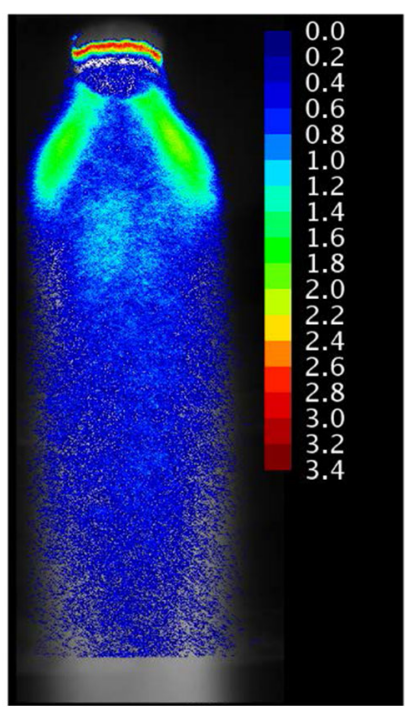

(b)
Fig. 3-Heat maps showing the logarithm (base 10) of (a) the number of positive and $(b)$ the number of negative displacement estimations generated by the computer vision algorithm (excluding displacement estimations equal to zero) (Color figure online).

recording of a three-dimensional process, there is no way of preventing errors that arise when clouds of particles move in front of, or behind, each other. In addition, the analysis method assumes that density gradient features move either towards or away from the nozzle. However, careful examination from one image to the next shows that clouds of particles are occasionally observed moving perpendicular to the main (vertical) direction of particle movement. Such horizontal motion is possible because there is an area of recirculation close to the nozzle and because highly turbulent flow conditions can also occur on a local level, causing localized horizontal motion. However, due to the low frequency of such occurrences, and to keep the complexity of the algorithm manageable, we have restricted tracking to the vertical direction. Although some false matches will therefore inevitably occur, by setting the analysis variables conservatively, the rate at which false matches occur can be minimized. Additionally, as a large number of features are identified and tracked, data arising from a small proportion of false matches will have only a small effect on the estimated mean velocities obtained.

The number of positive (downward) displacement estimations made at each location in the atomization plume is shown in Figure 3(a). From this heat map, it can be seen that the greatest number of displacement estimations are generated at the boundary between the nozzle, which is dark and stationary, and clouds of bright particles below the nozzle. This omnipresent density gradient typically generates displacement estimations on every pair of images that are analyzed, and so a very large number of displacement estimations are created at this location. As these displacement estimations are in part generated by a stationary piece of hardware (the nozzle), they are not considered to be genuine estimations of displacement, and so are removed from statistical analysis of the displacement frequency distribution. These are excluded by filtering the data to remove all displacement estimations that occur within the first 79 rows of pixels.

Having discounted the displacement estimations generated by the omnipresent density gradient at the nozzle edge, the greatest concentration of displacement estimations is seen to occur at the atomization plume shoulders, where the atomization plume increases from nozzle width to a maximum width. Upon viewing a sample of the individual images, this result is expected, because the shoulder area of the atomization plume frequently contains clearly defined density gradient features, with a high level of contrast existing between bright, highly dense clouds of particles and the black background above which is free of any atomized material.

Below the nozzle and between the shoulders is an area with relatively few displacement estimations. Inspection of a video compilation of the high-speed camera images shows that this more sparsely populated area corresponds to a region that often appears very bright, to the extent that the camera sensor was probably often at, or near, its saturation point. In an area where the majority of pixels are at, or near, saturation, it follows that few density gradient features will be identified. Further down the atomization plume the saturation of the camera sensor is reduced due to a greater dispersion of particles and cooling of the droplets reducing their brightness. Consequently, downstream conditions favor a higher number of detections. However, this assistance is offset somewhat by the more diffuse nature of the boundaries between areas of high and low particle density. This is due to particles becoming more evenly distributed in the gas stream as mixing due to turbulence begins to disperse the dense clouds of particles.

The number of negative (upward) displacement estimations made at each location in the atomization plume is shown in Figure 3(b). From this heat map, it can be seen that negative displacement estimations are most concentrated in the top third of the atomization plume, which equates to a distance of around 2 to 3 nozzle diameters from the melt outlet. This is the area that corresponds to the approximate extent of the recirculation zone, within which clouds of particles are sometimes observed moving towards, rather than away from the nozzle.

Data relating to the identification and tracking stages of the image analysis process are shown as heat maps in Figure 4. The average density gradient at each location (identification stage in image 1) is shown in Figure 4(a), while the average correlation coefficient at each location (tracking stage in image 2) is shown in Figure 4(b). The highest average density gradients are found on the shoulders of the plume. This is expected as inspection of the input images shows that the shoulder area of the plume is frequently occupied by highly dense clouds of particles, and when contrasted with the black background above, strong density gradients are produced. As distance from the nozzle increases, the average density gradient decreases in value. This reduction is due to increased dispersion of molten droplets, so that the boundaries between areas that are densely populated with particles and those areas that are more sparsely populated become more diffuse. Furthermore, as the 


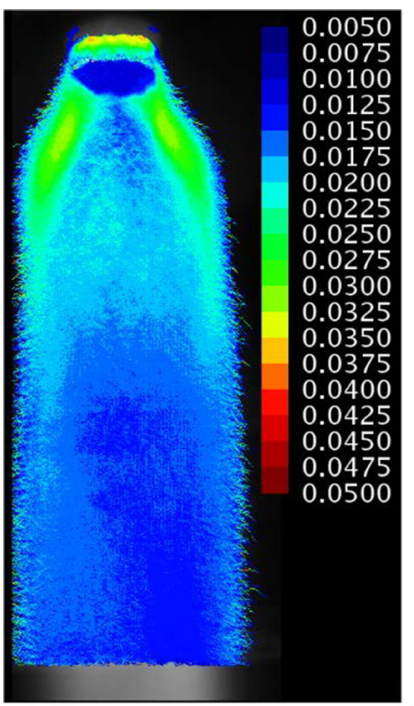

(a)

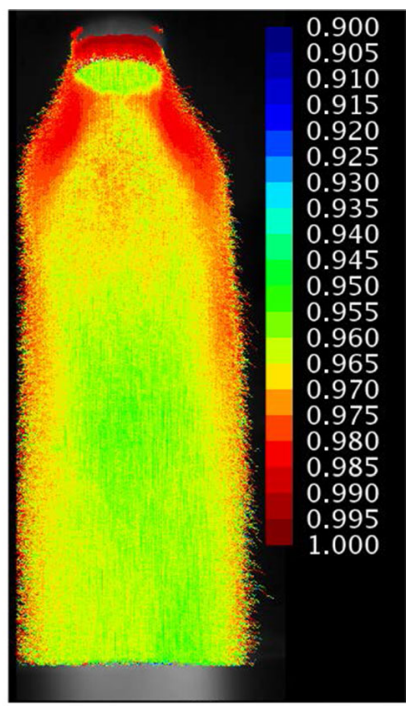

(b)
Fig. 4-(a) Average density gradient at each location for the identification stage of the computer vision analysis (image 1) and $(b)$ average correlation coefficient at each location for the tracking stage of the computer vision analysis (image 2) (Color figure online).

droplets cool, their radiance decreases, thereby reducing the potential for contrast and a strong density gradient between regions with differing concentrations of molten droplets. Immediately below the nozzle is an elliptical area with low average density gradient. This area is thought to be caused by frequent saturation of the camera sensor by dense clouds of very hot particles of molten metal. Some continuation of this effect is visible below the elliptical area, between the shoulders of the atomization plume. The average correlation values in the tracking stage have a similar pattern to that seen for the density gradient values in the identification stage. This is thought to be due to similar reasons as have been described for the average density gradient values (increasingly diffuse distribution of particles, and saturation of the camera sensor by dense, very hot clouds of molten metal particles).

\section{METHOD OF ESTIMATING KINETIC ENERGY WITHIN THE ATOMIZATION PLUME}

Having created spatial maps of the estimated velocity of atomized material in the plume, it is also possible to estimate the momentum and kinetic energy present within the plume of atomized material. This kind of analysis is useful, as if the momentum and kinetic energy supplied to the process by the atomizing gas is also estimated, then it is possible to quantify the efficiency with which momentum and kinetic energy is being transferred from the atomizing gas to the molten metal. The required mean (momentum) and RMS (kinetic energy) velocities are already known from the analysis. To make an estimation of the momentum and kinetic energy present within the plume, it is also necessary to make an estimate of how the mass is distributed within the melt plume.

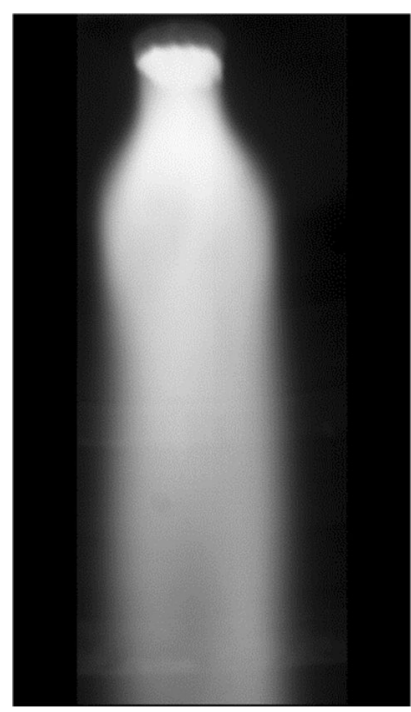

(a)

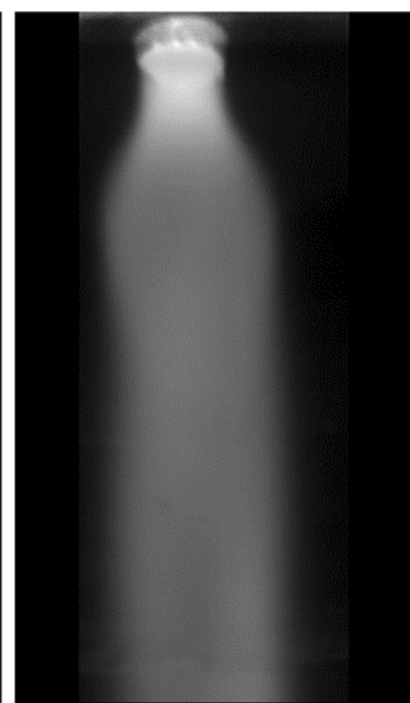

(b)
Fig. 5-Estimated distribution of relative mass within the atomization plume: (a) Average plume before applying correction $(b)$ Average plume after applying correction.

It is assumed that the mass of atomized material present at each pixel of the image is approximately proportional to the brightness (grayscale value) of that pixel. The grayscale value of each pixel was determined by taking the mean grayscale value for each pixel across all 28,665 frames. This operation created what can be described as an average image of the atomization plume, and the result is shown in Figure 5(a). As illumination for the high-speed photography comes solely from incandescence of the atomized material, and the atomized material is cooler, and therefore less bright, as displacement from the melt nozzle increases, a correction was applied to the grayscale values to account for this downstream cooling of the melt.

The correction was made by first calculating the sum of grayscale values for each row of the average atomization plume, and then determining a correction factor for each individual row so that the sum of grayscale values for each row was equal to the sum of grayscale values in every other row. In regions of the atomization plume where the density of atomized material was greatest (e.g., directly below the melt nozzle), application of these correction factors resulted in some pixels with a grayscale value greater than 100 pct (a value of 255 for 8-bit grayscale values or a value of 1 for double-precision floating-point grayscale values). Therefore, following application of the correction factors on a row-wise basis, the grayscale value of every pixel was normalized with respect to the maximum grayscale value in the corrected average atomization plume so that no pixel had a grayscale value greater than 100 pct. The result, which is shown in Figure 5(b), is an image in which the grayscale is now proportional to the relative amount of mass present within that pixel volume.

We compute the mass weighted mean and mean square velocity, $\bar{V}$ and $\overline{V^{2}}$, respectively, as 


$$
\bar{V}=\frac{\sum_{i, j} m_{i, j} V_{i, j} r_{i}}{\sum_{i, j} m_{i, j} r_{i}}
$$

and

$$
\overline{V^{2}}=\frac{\sum_{i, j} m_{i, j} V_{i, j}^{2} r_{i}}{\sum_{i, j} m_{i, j} r_{i}},
$$

where $i$ labels the pixel row and $j$ labels the pixel column, with $m_{i, j}$ being the relative mass present at pixel $(i, j)$. The factor $r_{i, j}$ is the distance from the center of the plume which is introduced to account for the cylindrical symmetry of the system, whereby more mass is contained the further out on the plume a pixel lies. As the actual, time-averaged mass discharge from the nozzle is known $\left(0.25 \mathrm{~kg} \mathrm{~s}^{-1}\right), \bar{V}$ and $\overline{V^{2}}$ can then be used to obtain the actual momentum and kinetic energy embodied within the plume by virtue of the motion of the melt.

We assume that the velocity, and hence momentum and kinetic energy, embodied within the supersonic gas stream can be estimated using isentropic flow theory with (near) ideal expansion. For a De Laval nozzle profile with outlet diameter $A$ and constriction (throat) area $A^{*}$, the Mach number, $M$, at ideal expansion is given by

$$
\frac{A}{A^{*}}=\frac{1}{M}\left(\frac{\gamma+1}{2}\right)^{-\kappa}\left(1+\frac{\gamma-1}{2} M^{2}\right)^{\kappa},
$$

where $\gamma$ is the ratio of specific heats $\left(c_{\mathrm{p}} / c_{\mathrm{v}}\right)$ and $\kappa=\frac{1}{2}(\gamma+1)(\gamma-1)$. This in turn may be related to the pressure requirement for ideal expansion, wherein

$$
\frac{P}{P_{0}}=\left(1+\frac{\gamma-1}{2} M^{2}\right)^{\frac{\gamma}{\gamma-1}},
$$

where $P$ is the pressure of the gas on the inlet side of the manifold and $P_{0}$ is the plenum pressure on the outlet side. For an inlet pressure of $2.60 \mathrm{MPa}$ and an outlet maintained at (or close to) atmospheric pressure, we have a Mach number upon exit of 2.778, corresponding to a design criterion of $A / A^{*}=2.896$ (for $\mathrm{N}_{2}$ gas as used in the atomizer used here, $\gamma=1.4$ for a diatomic species). The corresponding drop in temperature upon expansion is given by

$$
\frac{T_{0}}{T}=\left[1+\frac{\gamma-1}{2} M^{2}\right]^{-1},
$$

where $T_{0}$ is the temperature of the expanded gas in the plenum and $T$ that of the unexpanded gas in the manifold. Equation [5] gives $T_{0} / T$ as 0.4712 , wherein for an inlet temperature of $473 \mathrm{~K}$, as used in the atomizer here, we obtain an outlet temperature of $223 \mathrm{~K}$. The corresponding velocity of sound is $299 \mathrm{~m} \mathrm{~s}^{-1}$. Consequently, the linear velocity of the gas at exit is estimated at $831 \mathrm{~m} \mathrm{~s}^{-1}$.

\section{RESULTS}

Heat maps of the estimated average, RMS, 95th percentile, and 5th percentile velocity at each spatial location are shown in Figures 6(a) through (d), respectively. With respect to the average velocity (Figure 6(a)), the center of the atomization plume is seen to contain the lowest velocity estimations, surrounded by a skin of higher velocity material. This result agrees with video compilations of the high-speed camera images, in which clouds of particles can clearly be seen streaming away from the nozzle rapidly at the periphery of the atomization plume, while the interior appears, on average, to contain slower clouds of material. The skin of faster

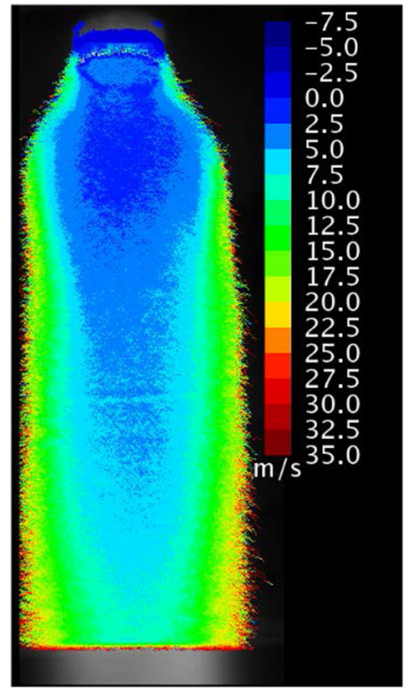

(a)

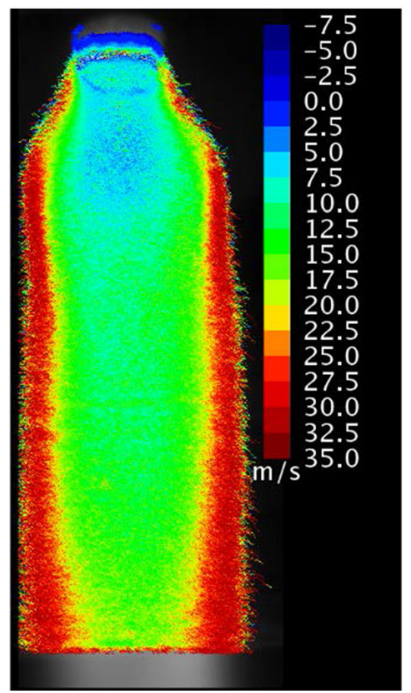

(c)

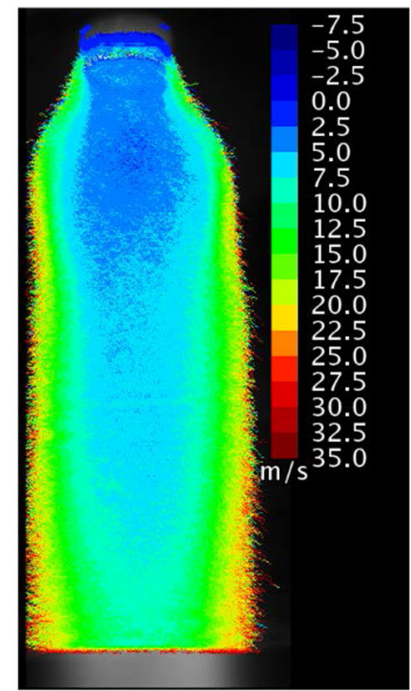

(b)

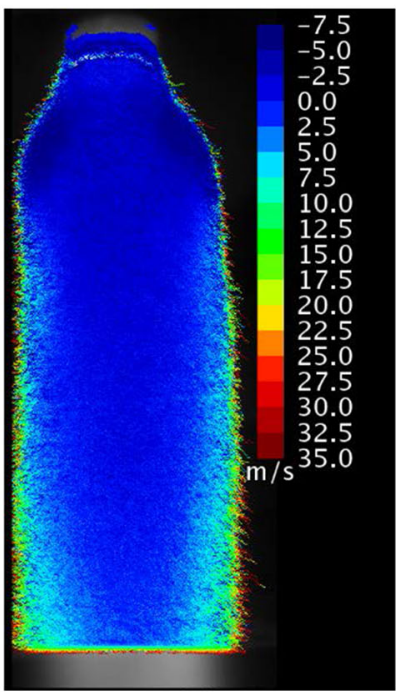

(d)
Fig. 6-(a) Average, (b) RMS, (c) 95th percentile, and (d) 5th percentile estimated velocity at each location in the atomization plume (Color figure online). 
moving particles is observed to thicken slightly as distance from the nozzle increases. This is as expected, as viscous shear forces in the gas will gradually transfer energy from the higher velocity periphery of the atomization plume to the lower velocity inner region. Of particular interest is the interior region in the topmost 25 to 33 pct of the atomization plume ( 2 to 3 nozzle diameters downstream from the melt outlet), in which it is observed that the plume contains the lowest velocity material, with the average velocity being close to, or less than, zero. This region corresponds to the widely discussed recirculation zone, whereby the negative (upward) velocity of the recirculating material reduces the average velocity of material in the plume to (close to) zero. Manual inspection of the movie from high-speed filming shows that there is indeed evidence of recirculation occurring in this area of the plume, in the form of clouds of particles observed moving towards the nozzle, giving us confidence in this result. The RMS velocity distribution (Figure 6(b)) is broadly similar, but with the low velocity region below the nozzle being less well defined. This is to be expected as the RMS process eliminates any negative (upwards) velocities present in the recirculation zone.

The 95th percentile estimated velocity heat map (Figure 6(c)) is similar to the average velocity heat map in that there is a lower velocity core of material surrounded by a higher velocity skin, with the lowest estimated velocities again being in the inner region of the topmost 25 to 33 pct of the atomization plume. In the 5 th percentile estimated velocity heat map (Figure 6(d)), negative velocity and low positive velocity estimations are seen to be present over the entire atomization plume area, although as can be seen from Figure 3(b), the number of detections of negative velocity is low in the bottom half of the atomization plume. The highest magnitude negative flow is found in the two lobes on the margin of the melt plume in the first 1 to 1.5 nozzle diameters downstream from the melt outlet. Some caution should be exercised here as this is the region in which the plume expands from nozzle width to its full lateral width, wherein sideways movement of an obliquely orientated feature will produce a negative velocity estimation. However, the large number of negative velocity detections (Figure 3(b)) in this region together with the high average correlation coefficient with which the matches are made (Figure 4(b)) led us to believe that the majority of these negative velocity matches are genuine. This view is confirmed by careful observation of the original high-speed video. Lower down in the atomization plume the flow of the melt is expected to be directed downwards, i.e., with positive velocity. Therefore, it is hypothesized that the small number (Figure 3(b)) of negative velocities seen in the 5th percentile estimated velocity heat map are due to localized turbulence in the atomization plume.

The recirculation zone is often depicted as a region of stable recirculation below the melt nozzle, with counter rotating cells that move downwards with the gas at the margin of the plume and return upwards in line with the melt nozzle bore. This then spreads radially outwards across the melt nozzle tip, thus giving rise to the

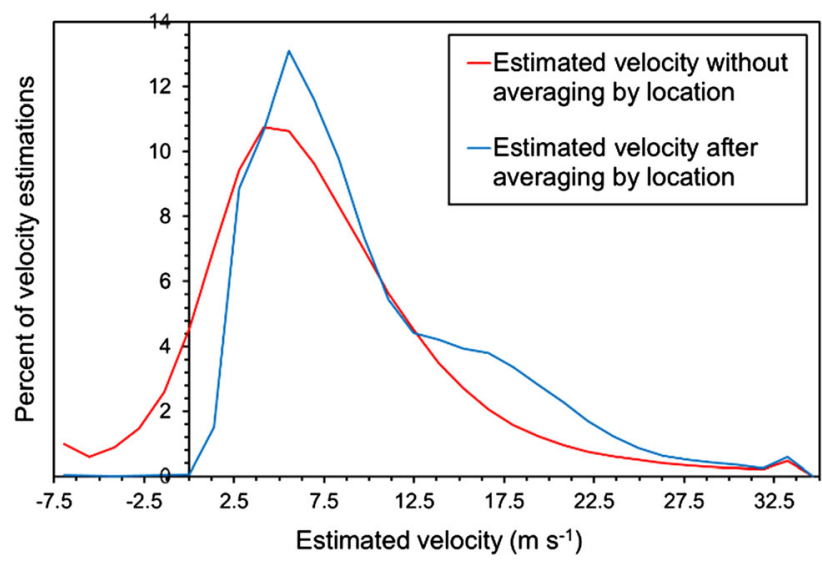

Fig. 7-Estimated velocity relative frequency distribution, with and without averaging by location (Color figure online).

important melt pre-filming. Such a view of the recirculation zone is supported by modeling studies such as those performed by Anderson and Ting, ${ }^{[23]}$ albeit in gas-only flow due to the computational difficulty in performing two-fluid simulations. However, neither the velocity data nor direct observation of the high-speed filming appears to support this view. A well-defined recirculation pattern would lead to a clear delineation between materials with a negative velocity below the central bore of the melt nozzle and material with a positive (downwards) velocity towards the margins of the recirculation zone. Instead, we see a region in which the average velocity is near zero across the recirculation zone, which in the video appears as a rather chaotic motion of the melt, sometimes directed with the gas flow direction, sometimes against it. Moreover, the regions of maximum negative velocity are the two lobes below and somewhat to the sides of the melt nozzle as the melt plume expands to full width.

To explore this further we plot in Figure 7 two relative frequency histograms. One of these is the distribution of all the individual velocity determinations made, while the other is the distribution after spatial averaging, that is it represents the distribution of the velocity magnitudes depicted in the heat map given in Figure 6(a). The spatially averaged curve is thus based upon 169,036 points, this being the number of unique locations at which at least one velocity estimation was made. By way of comparison, the curve for the non-averaged data is based upon $13,958,823$ points, this being the total number of velocity estimations made, as given in Table $\mathrm{C} 1$ (Appendix C). What is clear from the figure is that while $\sim 6.5$ pct of total number of velocity estimations are negative, the number of locations where the average velocity is negative is sufficiently small $(0.16 \mathrm{pct})$ as to be insignificant. This argues strongly against there being a stable recirculation pattern as observed in gas-only flow. If such a pattern were to be present over periods comparable to the filming interval (1.7916 seconds), the negative velocity of the upward moving plume would be expected to be observed in the average velocity map, and this is not 
the case. However, clouds of particles with a negative velocity have been observed moving in an upwards direction (i.e., towards the nozzle) over much shorter time periods, of the order of 12 frames, which equates to 0.75 milliseconds.

Consequently, we conclude that while recirculation certainly does occur during two-fluid atomization, this does not occur in the steady, well-defined manner observed in gas-only flow, being instead chaotic in nature. However, the size of the recirculation zone is broadly in line with estimates from computational modeling reported by Anderson and Ting, ${ }^{[23]}$ and Schlieren imaging studies under conditions of gas-only flow published by Mates and Settles. ${ }^{[15]}$ Expansion of the atomizing gas followed by recompression results in a high-pressure stagnation point, which is normally considered to be the termination of the recirculation zone. Typically this feature is placed around two nozzle diameters downstream from the melt outlet, a location which is consistent with the termination of very low average velocity in the Figure 6(a).

The other feature that is most striking in the velocity maps presented here is the relatively low velocity of the atomized particles, which even on the periphery of the melt spray cone is only of the order of $30 \mathrm{~m} \mathrm{~s}^{-1}$, and much less in the center of the spray cone. This result is consistent with the point estimate published by Mullis et al. ${ }^{[13]}$ for material within a high-pressure gas atomization plume, which was also of order $30 \mathrm{~m} \mathrm{~s}^{-1}$. In contrast, the gas used to break up the molten metal into droplets will be supersonic. For an inlet gas pressure of $2.6 \mathrm{MPa}$, isentropic flow theory would predict that the exit velocity of a diatomic gas at the jet will be Mach 2.78. At room temperature, this would equate to $\sim 930 \mathrm{~m} \mathrm{~s}^{-1}$, although under adiabatic expansion the gas would cool to around 0.48 of its pre-expansion absolute temperature, lowering the velocity of the gas, which has been pre-heated to $473 \mathrm{~K}$ prior to expansion, to around $831 \mathrm{~m} \mathrm{~s}^{-1}$. The large difference between gas velocity at the point of discharge and the velocity of molten metal droplets or clouds of molten metal droplets means that very little of the momentum and kinetic energy in the gas is transferred to the molten metal. With a gas mass flow of $0.35 \mathrm{~kg} \mathrm{~s}^{-1}$, the power and momentum flux in the gas stream are $120.8 \mathrm{~kW}$ and $290 \mathrm{~kg} \mathrm{~m} \mathrm{~s}^{-2}$. The mass flow rate for the melt is $0.25 \mathrm{~kg} \mathrm{~s}^{-1}$ and, with an RMS and mean velocity of 12.77 and $9.96 \mathrm{~m} \mathrm{~s}^{-1}$, respectively, the corresponding power and momentum flux in the melt are $20.4 \mathrm{~W}$ and $2.49 \mathrm{~kg} \mathrm{~m} \mathrm{~s}^{-2}$. Consequently, the efficiency of the atomizer in transferring momentum from the gas to the melt is estimated at $0.86 \mathrm{pct}$, while the corresponding efficiency in transferring kinetic energy from the gas to the melt is 0.017 pct. Interestingly, this latter value is of the same order as the theoretical efficiency for converting kinetic energy into embodied surface energy within the powder.

The results presented here shed considerable new light on the gas atomization process, and particularly on its low efficiency and wide particle size distribution. Even at the margin of the melt plume, where the melt is in direct contact with the supersonic gas, the velocity at which the melt is observed to stream away from the nozzle is only around 4 pct of that of the gas velocity. This in turn leads to a very low calculated efficiency for the transfer of kinetic energy from the gas to the melt, determined in this case as 0.017 pct. Moreover, given the distribution of velocities within the atomization plume, the wide spread in the PSD of powders produced by gas atomization can also be rationalized. In particular, much of the melt plume appears to be relatively well shielded from the gas, wherein we speculate that primary atomization on the margins of the plume leads to relatively fine ligaments being produced to feed into the secondary atomization zone. Conversely, much coarser ligaments would be produced in the interior of the plume, with these size differences persisting through secondary atomization.

\section{CONCLUSIONS}

A computer vision algorithm has been developed to process high-speed photography images of the gas atomization process for the purpose of estimating local velocities within the melt plume. The analysis method produces comparable velocity estimations for a wide range of tracking settings, thereby being demonstrably insensitive to the precise settings chosen for tracking. The velocity estimates thus obtained are consistent with the very limited such data obtained in previous studies. However, relative to the gas velocity, $\approx 831 \mathrm{~m} \mathrm{~s}^{-1}$, maximum velocities in the melt plume are very low $\left(<35 \mathrm{~m} \mathrm{~s}^{-1}\right)$. Consequently, the efficiency of kinetic energy transfer between gas and melt is estimated to be $<0.02$ pct. Moreover, the distribution of velocities within the plume is extremely heterogeneous, with the interior of the plume effectively being shielded from the gas by the melt on the plume margins, potentially accounting for the wide spread in the PSD of powders produced by gas atomization. In summary, we believe that the ability to produce spatially resolved velocity maps for the second fluid in a high-pressure gas atomization plume opens up a new avenue of research in gas atomization. Not only can such maps help explain common features of the powder size distribution of gas atomized powders, they give a direct means of quantifying the efficiency of the energy and momentum transfer in situ. In regard to this latter point, velocity mapping could also be used to assess the effect of design modifications in improving the efficiency of energy transfer between the gas and the melt.

As an aside we also note that the velocity determination routines developed here could have wider application to other types of atomization processes in which dense clouds of particles are formed. One such application would be to spray drying. As far as we can envisage, depending upon the illumination used, the only potential change is that we would need to search for dark clouds of particles against a lighter background, wherein we would be searching for a lightto-dark density gradient to detect the leading edge of a cluster of particles, rather than a dark-to-light density gradient. 


\section{ACKNOWLEDGMENTS}

This work was funded by MAPP - The EPSRC Future Manufacturing $\mathrm{Hub}$ in Manufacture using Advanced Powder Processes (Grant No. EP/P006566/ 1). It was enabled by the use of high-speed camera images obtained from a gas atomizer owned and operated by Atomising Systems Limited, Sheffield.

\section{CONFLICT OF INTEREST}

All authors declare that they have no conflict of interest.

\section{OPEN ACCESS}

This article is licensed under a Creative Commons Attribution 4.0 International License, which permits use, sharing, adaptation, distribution and reproduction in any medium or format, as long as you give appropriate credit to the original author(s) and the source, provide a link to the Creative Commons licence, and indicate if changes were made. The images or other third party material in this article are included in the article's Creative Commons licence, unless indicated otherwise in a credit line to the material. If material is not included in the article's Creative Commons licence and your intended use is not permitted by statutory regulation or exceeds the permitted use, you will need to obtain permission directly from the copyright holder. To view a copy of this licence, visit http://creativec ommons.org/licenses/by/4.0/.

\section{ROLE OF THE FUNDING SOURCE AND INDUSTRIAL SPONSOR}

Neither the funding source nor Atomising Systems Limited had any role in the development of the computer vision algorithm, the interpretation and analysis of data resulting from the computer vision algorithm, or the writing of this report.

\section{APPENDIX A: DETAILED, TECHNICAL DESCRIPTION OF THE COMPUTER VISION ALGORITHM}

The images from the high-speed camera are considered to consist of a matrix of grayscale values with a width of $i$ width and a height of $i$ height. The location of each pixel is denoted by a row index of $j_{1}$ and a column index of $j_{2}$, using the standard matrix notation of labeling rows first (from top downwards) followed by columns (from left to right). Starting from the first frame in the sequence, pairs of images, which in general we will denote as frames $N$ and $N+1$, are analyzed to identify dominant features in frame $N$ and track these to frame $N+1$, in order to determine the displacement between frames. Starting in the top left corner of image
$N$, at pixel $j_{1}=1$ and $j_{2}=1$, a one pixel wide vertical window of height win_size is assessed. The maximum and minimum grayscale values of the pixels within this window are identified and, if the difference between the maximum and minimum is greater than min_dens_diff, the grayscale density gradient within the window is computed using a linear regression function. If the grayscale density gradient within this vertical window exceeds a threshold value, slope_thresh, then a search is initiated, advancing the vertical window down the image, one pixel at a time, a distance of peak_search_win, to find the location at which the grayscale density gradient is at a local maximum. Once this search has been completed, the $\left(j_{1}, j_{2}\right)$ coordinate of the location of that maximum is stored.

The entire column is processed in this manner, advancing downwards (to a maximum of $j_{1}=i_{-}$height - (peak_search_win + win_size), searching for grayscale density gradients. When a density gradient that meets the min_dens_diff and slope_thresh requirements is found, we then search for and store the location of its local maximum value. The next column is then processed, and the process of searching for these local maxima repeated until the column $i_{-}$width is reached. In Figure 1(a), all of the local maxima in the grayscale density gradient identified by the computer vision algorithm in the image are highlighted (the highlighting width in this and following figures has been tripled from one pixel to three pixels wide, this is for illustrative purposes only).

For all the locations in image $N$ where a grayscale density gradient meets the search criteria, a search is initiated in image $N+1$ for a grayscale density gradient similar to that found in image $N$. The search starts in the same location as the local maximum found in image $N$, and extends upwards in the same column for a distance of upwa_search_win and downwards in the same column for a distance of down_search_win. If a negative density gradient (i.e., light to dark) is encountered that is less than neg_slope_thresh, then it is assumed that a better match will not be found. In that case, if the search is currently proceeding in an upwards direction, the direction is switched to downwards, and if the search direction is currently downwards, the search will end.

During the search for the best match in image $N+1$, the quality of the match to the grayscale density gradient found in image $N$ is evaluated at each search location. Two methods have been evaluated to do this. For the first option, a correlation coefficient between the window of pixels in image $N$ and the potentially matching window of pixels in image $N+1$ is computed. A match is not considered to have been made unless the correlation coefficient exceeds the threshold value of corrcoeff_thresh. Subject to exceeding this threshold, and the search being within the bounds of upwa_search_win and down_search_win, the location of the window in image $N+\overline{1}$ with the highest correlation coefficient to the corresponding window in image $N$ is stored as the best match. For the second option, the process of finding the best match is similar, but with slightly different criteria. Instead of assessing the quality of match by calculating a correlation coefficient, the grayscale density gradient at 
each location is calculated exactly as was done in image $N$. A match in image $N+1$ is not considered to have been made unless the grayscale density gradient found in image $N+1$ is within a defined tolerance, max_diff, of the grayscale density gradient found in image $N$. Results using both methods are very similar, which gives us confidence that features within the atomization plume are being correctly identified and tracked. By manually checking a large number of images and the associated matches made by the computer vision algorithm, it appears that the correlation method generates significantly more matches without adversely impacting robustness of the tracking process. Therefore, the correlation method has been adopted for all of the results presented here. Figure 1(b) shows an image $N+1$, with highlighting to indicate the tracked locations of the grayscale density gradients previously found in image $N$, and shown in Figure 1(a).

Grayscale density gradient features used to estimate velocity should preferably be prominent, so as to have a high probability of persisting from image $N$ to image $N+1$, thereby improving the robustness of the results obtained and reducing false matches in image $N+1$. However, the method described above sometimes identifies grayscale density gradient features that are only a single pixel wide or a few pixels in width. Using data from density gradient features that are only a few pixels

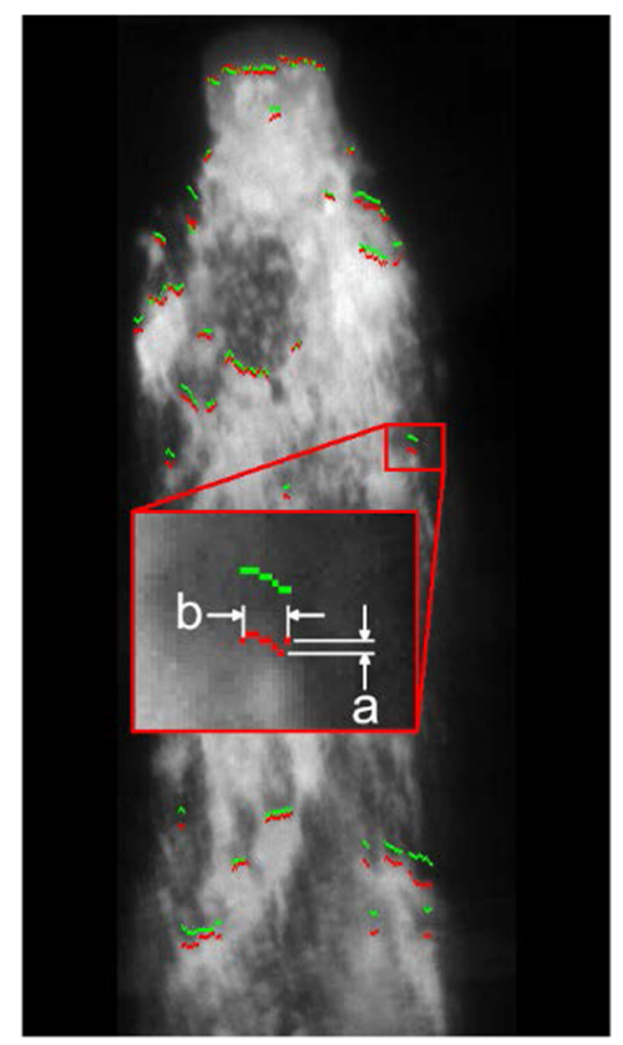

Fig. A1-An example of the max_vert_step (a) and min_len (b) parameters being applied to a density gradient feature. The location of the density gradient feature in frame $N$ is highlighted in green, while the new tracked location in frame $N+1$ is highlighted in red. The max_vert_step parameter is shown being applied to the two right-hand most density gradients from frame $N+1$ (Color figure online). wide is considered likely to reduce the robustness of the analysis. Therefore, once the identification and tracking processes have been completed on an image pair, we include a filtering process that removes identified features that are below a minimum length, min_len. Moreover, identified features are also sometimes found to contain significant vertical steps along the length of the feature. To ensure that features used for velocity estimation are reasonably continuous, filtering is employed to restrict the maximum vertical step, max_vert_step, between any given pixel and the neighboring pixel in the next column of the same feature. Figure A1 shows an example of the parameters min_len and max_vert_step being applied to density gradients. For features that are sufficiently long, this type of filtering means that where there are vertical steps that exceed max_vert_step, the feature will be broken up into two or more sections, and any sections below min_len will then be discarded. In Figures 2(a) and (b), the filtered data from Figures 1(a) and (b) are shown, from which it can be seen that some of the features that were identified and tracked have been excluded. Figure 2(a) shows image $N$, while Figure 2(b) shows image $N+1$. In both images, the locations of density gradient features identified in image $N$ are highlighted in green, while the locations of density gradient features tracked in image $N+1$ are highlighted in red. When the computer vision algorithm has analyzed a pair of images, $N$ and $N+1$, the process is iterated, with image $N+1$ becoming image $N$, until all of the images available have been analyzed.

\section{APPENDIX B: DESCRIPTION OF THE COMPUTER VISION ALGORITHM PARAMETERS}

$i_{\text {_height }}$ and $i_{-}$width - These parameters are the height and width, in units of pixels, of the images to be processed by the computer vision algorithm.

win_size - This is the height, in units of pixels, of the vertical window over which the computer vision algorithm assesses the grayscale density gradient to determine if a potentially trackable density gradient exists at location $j_{1}, j_{2}$ within image $N$. The vertical window is always one pixel wide, and so there is no setting for window width.

min_dens_diff - This is the minimum grayscale density difference between the brightest pixel and the darkest pixel in the vertical window when searching for trackable grayscale density gradients in image $N$. If this difference is exceeded and the minimum value for grayscale density gradient (slope_thresh) is also exceeded, then a search is initiated for a local grayscale density gradient maximum.

slope_thresh-This is the minimum value for grayscale density gradient when searching for trackable grayscale density gradients in image $N$. If the setting for this parameter is exceeded and the minimum value for grayscale density difference (min_dens_diff) is also exceeded, then a search is initiated for a local grayscale density gradient maximum. 
peak_search_win - Once the existence of a grayscale density gradient in image $N$ has been determined (via min_dens_diff and slope thresh), this parameter is the distance, in units of pixels, to search for the local maximum of that grayscale density gradient. As the search for trackable grayscale density gradients proceeds from the top to the bottom of each column in image $N$, the maximum distance to search, peak_search_win, is applied in a downwards direction from the location where the min_dens_diff and slope_thresh parameters were first satisfied.

upwa_search_win - This parameter is the distance, in units of pixels, to search upwards (towards the melt nozzle) for a matching grayscale density gradient in image $N+1$. The search for a matching grayscale density gradient in image $N+1$ commences at the $j_{1}, j_{2}$ location where the grayscale density gradient was recorded to be at a local maximum in image $N$. Allowing the computer vision algorithm to search upwards as well as downwards allows for the fact that a small recirculation zone exists close to the melt nozzle, where clouds of atomized material are sometimes observed moving towards, rather than away from the melt nozzle.

down_search_win - This parameter is the distance, in units of pixels, to search downwards (away from the melt nozzle) for a matching grayscale density gradient in image $N+1$. This parameter is similar to parameter upwa_search_win, in that it constrains the distance that the computer vision algorithm can search for a matching grayscale density gradient in image $N+1$.

neg_slope_thresh - When searching for trackable grayscale density gradients in image $N$, the computer vision algorithm searches for positive gradients (dark to light). Therefore, if a negative density gradient (light to dark) is encountered when searching for a matching grayscale density gradient in image $N+1$, it is assumed that continuing with the search will not yield a match, or if a match has already been encountered, a better match will not be found. Therefore, in the case that the search direction is proceeding in an upwards direction towards the limit specified by upwa search win, the search direction is switched to downwards. If the search direction is proceeding in a downwards direction when a negative grayscale density gradient is encountered, then the search for a matching grayscale density gradient is terminated.

corr_coeff_thresh - When the computer vision algorithm is set to find matches in image $N+1$ using correlation coefficient as the matching method, this parameter is used to set the minimum correlation coefficient between the grayscale density gradient found in image $N$ and the grayscale density gradient found in image $N+1$. If the correlation coefficient between the grayscale density gradients found in image $N$ and $N+1$ does not exceed the value of corr_coeff_thresh, then the grayscale density gradient found in image $N+1$ is not recorded as a match.

max diff - When the computer vision algorithm is set to find matches in image $N+1$ using grayscale density gradient as the matching criteria, max_diff is the maximum permissible difference between the grayscale density gradient found in image $N$ and the grayscale density gradient found in image $N+1$. For example, when max_diff is set to a value of 0.25 , if the grayscale density gradient found in image $N+1$ is not within \pm 25 pct of the grayscale density gradient found in image $N$, then a match is not recorded. Note that the use of max_diff is an alternative method for finding matches in image $N+1$, and the results presented herein were derived from matches found using corr_coeff_thresh.

max_vert_step - This parameter is used in the filtering stage of the computer vision algorithm to improve the robustness of the results by identifying grayscale density gradient features that are discontinuous. Discontinuous density gradient features are eliminated by searching in the column to the right of each density gradient match found in image $N+1$ to ascertain if another density gradient has been recorded in close proximity. The max_vert_step parameter is used to set the vertical distance, $\overline{\text { in }}$ pixels, that the computer vision algorithm checks upwards and downwards in the next column to determine if another density gradient has been recorded in close proximity. The computer vision algorithm keeps a $\log$ of those density gradients that are part of a continuous density gradient feature.

min_len - Once the computer vision algorithm has determined which density gradients are part of a continuous density gradient feature (see description of the max_vert_step parameter), and the horizontal width, in pixels, that each continuous density gradient feature spans, the parameter min_len is used to filter the density gradients. All density gradients that are not part of a density gradient feature that is at least min_len pixels wide are discarded. This improves the robustness of the computer vision algorithm by removing small density gradient features that are only a single pixel wide or span only a few pixels in width.

\section{APPENDIX C: METHODOLOGY FOR SETTING THE UPWA SEARCH WIN AND DOWN_SEARCH_WIN PĀRAMETERS}

One of the key parameters is the search range used to track features. Changing the settings for upwa_search_win and down_search_win has the effect of varying the range over which the computer vision algorithm is able to search for matching density gradients in image $N+1$. In Figure $\mathrm{C} 1(\mathrm{a})$, the relative frequency distribution of the displacement estimations is presented for different search range settings in image $N+1$. The datasets have been filtered to remove any displacement estimations generated in the first 79 rows of pixels. Filtering was necessary to exclude displacement estimations resulting from the omnipresent density gradient generated by the edge of the nozzle. From these datasets, it can be seen that the relative frequency distributions obtained are very similar, even when the settings used for the tracking stage of the image analysis process are significantly different. The lack of sensitivity to the settings used in the tracking stage of the analysis process gives confidence that the computer vision algorithm produces 


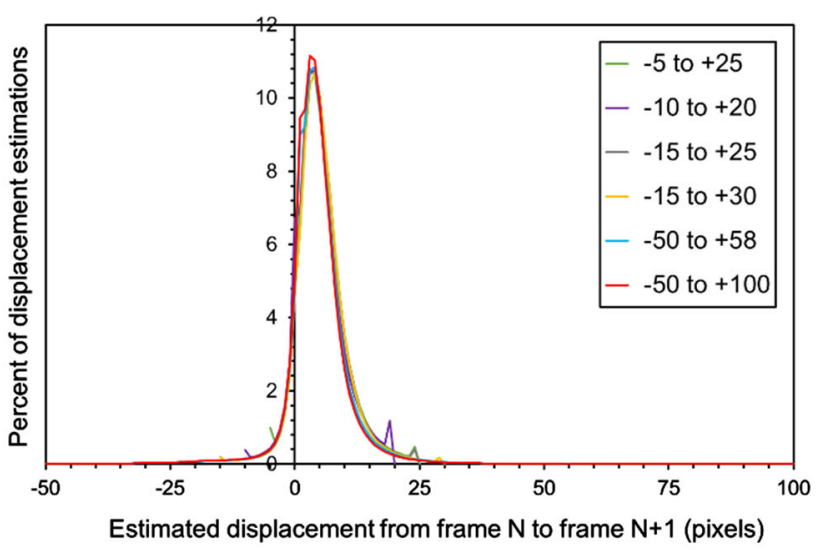

(a)

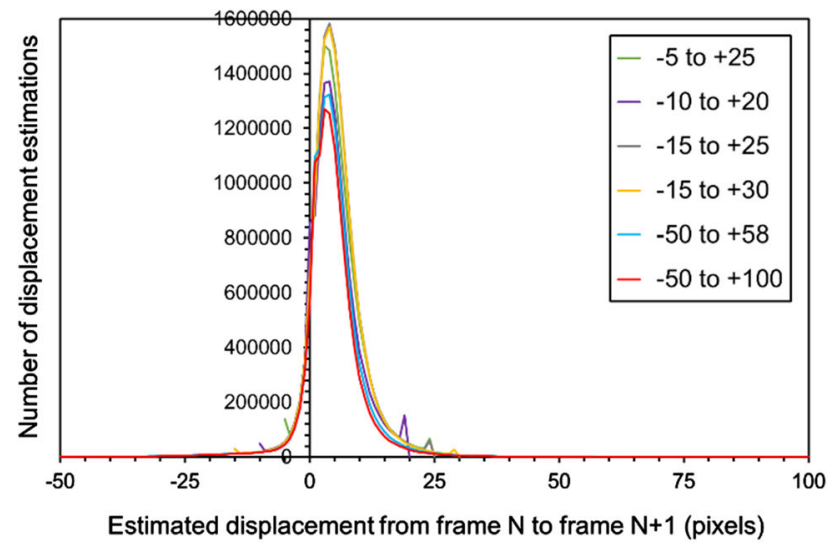

(b)

Fig. C1-(a) Relative and (b) absolute frequency distribution of displacement estimations using different allowable search ranges in image $N+1$ (Color figure online).

comparable results when these settings are varied and is therefore able to track density gradients in a way that is reasonably independent of the precise tracking settings used.

At the maximum and minimum estimated displacements, the relative frequency distributions show a slight increase. This result is seen in all of the relative frequency distributions, although in some cases the extremely low frequencies at the maximum and minimum estimated displacements conceal its presence. The slight increase in detection frequency at the limits of the search window consists of two types of match. Firstly, there are instances where the match criteria are met (minimum correlation coefficient or within the allowed tolerance for gradient matching), but the genuine best match is located outside the range of displacements where the computer vision algorithm is permitted to search. Secondly, if the density gradient found in image $N$ dissipates or disperses, and so is not present in image $N+1$, a false match may be found that meets the criteria (minimum correlation coefficient or within the allowed tolerance for gradient matching). However, if the best false match is located outside the search range, the observed spike in detections at the limit of the search window will be generated.
Table C1. Number of Displacement Estimations Resulting from Different Allowable Search Ranges (Excluding Displacement Estimations from the First 79 Rows of Pixels)

Allowable Search Range in Total Number of DisplaceImage $N+1 \quad$ ment Estimations

\begin{tabular}{ll}
\hline-5 to +25 Pixels & 13958823 \\
-10 to +20 Pixels & 12766277 \\
-15 to +25 Pixels & 14840837 \\
-15 to +30 Pixels & 14711192 \\
-50 to +58 Pixels & 12210456 \\
-50 to +100 Pixels & 11376766 \\
\hline
\end{tabular}

In either of these situations, a small spike in matches would be expected at the maximum and minimum estimated displacements. When the allowable search range is set with relatively narrow limits, the first scenario would best explain the spike in matches at the maximum and minimum estimated displacements. When the allowable search range is set with relatively wide limits, the second scenario would best explain the spike in matches at the maximum and minimum estimated displacements. As it is necessary to set an allowable search range for the tracking stage, it is considered that this type of response can never be totally eliminated when using a computer vision algorithm of this design to extract displacement estimations from high-speed camera images. It is important that the allowable search range is set correctly, so that genuine matches are not missed entirely or mislocated by a few pixels, and so that false matches to unrelated density gradients are minimized. This is achieved by manually inspecting the output images (e.g., Figure 2) and adjusting the computer vision algorithm's density gradient identification and tracking settings as appropriate.

In Figure $\mathrm{C} 1(\mathrm{~b})$, the dataset presented in Figure $\mathrm{Cl}$ (a) is replotted as an un-normalized frequency distribution to indicate the numbers of displacement estimations made for different tracking settings. As before, displacement estimations generated in the first 79 rows of pixels have been filtered out. When the data are plotted in this un-normalized form, it can be seen that by allowing the computer vision algorithm the greatest freedom $(-50$ to +100 range) in the tracking phase of the image analysis process, the peak is reduced in height. In Table $\mathrm{C}$, the total number of displacement estimations resulting from different tracking settings (different allowable search ranges) is shown. From the data in Table $\mathrm{Cl}$, it is apparent that allowing the computer vision algorithm to search a greater range of displacements in the tracking stage of the process does not necessarily result in a greater number of displacement estimations. Indeed, generally the trend is for fewer detections as the search range is extended in image $N+1$.

At first it may seem counterintuitive that allowing the computer vision algorithm a significantly greater degree of freedom in the tracking stage of image analysis can lead to fewer displacement estimations. However, when the analysis process is considered in its entirety, an explanation becomes apparent. By allowing the 
computer vision algorithm greater freedom, the best matches to the density gradient may be distributed over a wider range of displacements (the best matches may also include false matches). Subsequently, in the filtering stage of the analysis, the vertical step constraint applies. When the vertical step constraint is applied, if the matches for neighboring density gradients in image $N+1$ are distributed over a greater vertical range than the step constraint allows, then the density gradient feature will be broken up into two or more sections. A minimum length constraint also applies in the filtering stage of the analysis. Consequently, if the step constraint results in the density gradient feature being broken up into two or more sections, any section that falls short of the minimum length constraint will be discarded, thereby reducing the total number of displacement estimations in the analysis output.

Inspecting the output images (see Figures 2(a) and (b) for an example of output images) is an extremely labor-intensive task, and so it has not been possible to manually assess all of the image pairs for the presence of genuine and false matches. However, after checking a sample of output images, it appears that genuine matches in image $N+1$ occur in the approximate displacement range of -5 to +25 pixels from the density gradient feature originally identified in image $N$. Therefore, in the results and analysis that are presented, data are shown for image analysis settings in which the computer vision algorithm was constrained to the range of -5 to +25 pixels when searching for matches in image $N+1$.

\section{APPENDIX D: METHODOLOGY FOR SETTING THE COMPUTER VISION ALGORITHM PARAMETERS (EXCLUDING PARAMETERS UPWA_SEARCH_WIN AND DOW $\left.N_{-} S E A R \bar{C} H_{-} W I N\right)$}

\footnotetext{
i_height and $i_{-}$width - These parameters are both set according to the size of the images captured by the high-speed camera. In order to minimize the time required for the computer vision algorithm to process the images, it is advantageous to crop images beforehand if necessary, so that they do not contain dark areas with no information relating to the atomization process.

win_size - This parameter was set after inspecting a sample of the images to determine the approximate vertical scale and vertical spacing of the clouds of atomized material from which it was hoped to extract displacement and velocity information. The parameter was set so that the size of the window was large enough to identify a typical dark-to-light transition, but not so large as to span a dark-light-dark transition. Testing using different win size values was then undertaken to ensure that the setting was effective in identifying the density gradient features that are typically present.

min_dens_diff and slope_thresh - These parameters were set by experimentation with a range of different settings, and in conjunction with choosing an appropriate setting for the win_size parameter. In setting the values for these parameters, it was necessary to find a
}

value that identified as many density gradients as possible, while minimizing the false detections that would result from a setting that was too sensitive.

peak_search_win-This parameter was set using similar considerations to those used to set the parameter win_size.

upwa_search_win and down_search_win - The choice of settings used for these parameters is discussed in detail within Appendix C.

neg_slope_thresh - The main function of this parameter is to reduce the time taken for the computer vision algorithm to process the images, by curtailing the search for a matching density gradient in image $N+1$ when a match is unlikely to be found or a match better than the best match already identified is unlikely to be found. It was considered that provided the value was negative, the setting of this parameter would most likely have very little impact on the results obtained. Consequently, the value of this parameter was set to a small negative value without trialing a range of values.

corr coeff thresh and max diff - These parameters were set by experimenting with different values of corr_coeff_thresh and max_diff and inspecting the graphical outputs showing the locations of matching density gradients identified in image $N+1$.

max_vert_step and min_len - These parameters were set to optimize the robustness of the computer vision algorithm through elimination of discontinuous density gradient features and density gradient features that are only a few pixels wide. The parameter values were chosen by inspecting the graphical outputs showing the locations of density gradient features prior to any filtering. The effect of the filtering process using parameters max_vert_step and min_len can be seen by comparing Figures 1(a) and (b) (pre-filtering) with Figures 2(a) and (b) (post-filtering).

\section{APPENDIX E: THE SETTINGS USED FOR THE COMPUTER VISION ALGORITHM PARAMETERS}

See Table E1.

Table E1. Values of Image Analysis Parameters Used in the Computer Vision Algorithm (See Also Appendix F)

\begin{tabular}{ll}
\hline Image Analysis Variable & Value Used \\
\hline i_height & 800 \\
i_width & 312 \\
win_size & 26 \\
min_dens_diff & 0.2 \\
slope_thresh & 0.005 \\
peak_search_win & 30 \\
upwa_search_win & 5 \\
down_search_win & 25 \\
neg_slope_thresh & -0.001 \\
corr_coeff_thresh & 0.9 \\
max_diff & 0.25 \\
min_len & 5 \\
max_vert_step & 3 \\
\hline
\end{tabular}




\section{APPENDIX F: NOTE REGARDING GRAYSCALE VALUES AND THE SETTING OF PARAMETERS}

The high-speed camera used for this research records at a grayscale resolution of 8 bits, yielding integer values of 0 to 255 , where 0 corresponds to the darkest possible grayscale value and 255 corresponds to the lightest possible grayscale value. In the computer vision algorithm, pixel grayscale values are read in and processed as double-precision floating-point values. An 8-bit integer value of 0 is equal to a floating-point value of 0 , and an 8-bit integer value of 255 is equal to a floating-point value of 1 . Therefore, setting the min_dens_diff parameter to a floating-point grayscale value of $0 . \overline{2}$ corresponds to a minimum 8 -bit integer grayscale difference of 51 between the darkest and lightest pixels in the vertical window. Similarly, setting of the slope_thresh parameter to a floating-point value of 0.005 corresponds to a slope that is the equivalent of an 8-bit integer grayscale density difference of approximately 33 over a displacement (win_size) of 26 pixels.

\section{REFERENCES}

1. I.E. Anderson, E.M.H. White, and R. Dehoff: Curr. Opin. Solid State Mater. Sci., 2018, vol. 22, pp. 8-15.

2. I.E. Anderson and R.L. Terpstra: Mater. Sci. Eng. A, 2002, vol. 326, pp. 101-109.

3. D. Duke, D. Honnery, and J. Soria: Exp. Fluids, 2010, vol. 49, pp. $435-45$.

4. A. Zandian, W.S. Sirignano, and F. Hussain: In The 54th AIAA Aerospace Sciences Meeting (AIAA Proceedings: 2016), pp. 1-41.

5. X.-G. Li and U. Fritsching: J. Mater. Process. Technol., 2017, vol. 239, pp. 1-17.
6. J.M.C. Azevedo, A.C. Serrenho, and J.M. Allwood: Powder Technol., 2018, vol. 328, pp. 329-36.

7. A.J. Yule and J.J. Dunkley: Atomization of Melts for Powder Production And Spray Deposition, Oxford Series on Advanced Manufacturing No. 11, Clarendon Press, Oxford, 1994, pp. 22-25.

8. J. T. Strauss, S. A. Miller, In PM2TEC, International Conference on Powder Metallurgy \& Particulate Materials, Advances in powder metallurgy and particulate materials, 2001, pp. 2-28.

9. N.J. Grant: Atomization Die and Method for Atomizing Molten Material, 1984, United States Patent 4485834.

10. I.E. Anderson, R.L. Terpstra, and R.S. Figliola: Adv. Powder Metall. Particulate Mater., 2004, vol. 2, p. 26.

11. S. Motaman, A.M. Mullis, R.F. Cochrane, and D.J. Borman: Metall. Mater. Trans. B, 2015, vol. 46, pp. 1990-2004.

12. J.J. Dunkley, H. Danninger, and R. Ratzi: In Euro PM2004 (Euro PM2004 Proceedings: 2004), pp. 13-18.

13. A.M. Mullis, N.E. Adkins, Z. Aslam, I.N. McCarthy, and R.F. Cochrane: Int. J. Powder Metall., 2008, vol. 44, pp. 55-64.

14. A.M. Mullis, I.N. McCarthy, R.F. Cochrane, and N.J. Adkins: Metall. Mater. Trans. B, 2013, vol. 44, pp. 789-93.

15. S.P. Mates and G.S. Settles: Atomization Sprays, 2005, vol. 15, pp. 19-40.

16. Y. Ejiri, N. Hamada: In TENCON 2006 - IEEE Region 10 Conference (TENCON 2006 Proceedings: 2006).

17. T. Malavika and M. Poornima: Int. J. Eng. Res. Technol., 2013, vol. 2, pp. 1-4.

18. A.M. Mullis, I.N. McCarthy, and R.F. Cochrane: J. Mater. Process. Technol., 2011, vol. 211, pp. 1471-77.

19. C. Kirmse and H. Chaves: Steel Research International, 2016, vol. 87 , pp. $1295-1301$

20. M.P. Planche, O. Khatim, L. Dembinski, C. Coddet, L. Girardot, and Y. Bailly: Powder Technology, 2012, vol. 229, pp. 191-98.

21. P.X. Pham, A. Kourmatzis, and A.R. Masri: Exp. Thermal Fluid Sci., 2018, vol. 95, pp. 44-51.

22. P.T. Tokumaru and P.E. Dimotakis: Exp. Fluids, 1995, vol. 19, pp. $1-15$.

23. I.E. Anderson and J. Ting: Mater. Sci. Eng. A, 2004, vol. 379, pp. 264-78.

Publisher's Note Springer Nature remains neutral with regard to jurisdictional claims in published maps and institutional affiliations. 